\title{
Interface structure between tetraglyme and graphite
}

\section{$\operatorname{AUTHOR}(S)$ :}

Minato, Taketoshi; Araki, Yuki; Umeda, Kenichi; Yamanaka, Toshiro; Okazaki, Ken-ichi; Onishi, Hiroshi; Abe, Takeshi; Ogumi, Zempachi

\section{CITATION:}

Minato, Taketoshi ... [et al]. Interface structure between tetraglyme and graphite. The Journal of Chemical Physics 2017, 147(12): 124701.

\section{ISSUE DATE:}

2017-09-25

URL:

http://hdl.handle.net/2433/229001

\section{RIGHT:}

(C) 2017 AlP Publishing. This article may be downloaded for personal use only. Any other use requires prior permission of the author and AIP Publishing. The following article appeared in 'The Journal of Chemical Physics 147, 124701 (2017)' and may be found at http://aip.scitation.org/doi/10.1063/1.4996226.; The full-text file will be made open to the public on 25 September 2018 accordance with publisher's 'Terms and Conditions for Self-Archiving'. 


\section{Interface structure between tetraglyme and graphite}

Taketoshi Minato, Yuki Araki, Kenichi Umeda, Toshiro Yamanaka, Ken-ichi Okazaki, Hiroshi Onishi, Takeshi Abe, and Zempachi Ogumi

Citation: The Journal of Chemical Physics 147, 124701 (2017);

View online: https://doi.org/10.1063/1.4996226

View Table of Contents: http://aip.scitation.org/toc/jcp/147/12

Published by the American Institute of Physics

\section{Articles you may be interested in}

Researchers observe detailed structure of a model interface for a rechargeable battery

Scilight 2017, 140002 (2017); 10.1063/1.5004978

Single molecule translocation in smectics illustrates the challenge for time-mapping in simulations on multiple scales

The Journal of Chemical Physics 147, 114501 (2017); 10.1063/1.5001482

Phase and vacancy behaviour of hard "slanted" cubes

The Journal of Chemical Physics 147, 124501 (2017); 10.1063/1.5001483

Non-local effects of point mutations on the stability of a protein module

The Journal of Chemical Physics 147, 105101 (2017); 10.1063/1.4999703

Communication: Truncated non-bonded potentials can yield unphysical behavior in molecular dynamics simulations of interfaces

The Journal of Chemical Physics 147, 121102 (2017); 10.1063/1.4997698

Removing the barrier to the calculation of activation energies: Diffusion coefficients and reorientation times in liquid water

The Journal of Chemical Physics 147, 134103 (2017); 10.1063/1.4997723

\section{AIP $\mid$ The Journal of AIP $\mid$ Chemical Physics} PERSPECTIVES 


\title{
Interface structure between tetraglyme and graphite
}

\author{
Taketoshi Minato, ${ }^{1, a)}$ Yuki Araki, ${ }^{2,3}$ Kenichi Umeda, ${ }^{4}$ Toshiro Yamanaka, ${ }^{1}$ \\ Ken-ichi Okazaki, ${ }^{5}$ Hiroshi Onishi, ${ }^{2}$ Takeshi Abe, ${ }^{6}$ and Zempachi Ogumi ${ }^{5}$ \\ ${ }^{1}$ Office of Society-Academia Collaboration for Innovation, Kyoto University, Katsura, Nishikyo, \\ Kyoto 615-8510, Japan \\ ${ }^{2}$ Department of Chemistry, School of Science, Kobe University, Nada-ku, Kobe 657-8501, Japan \\ ${ }^{3}$ Department of Electronic Science and Engineering, Kyoto University, Katsura, Nishikyo, \\ Kyoto 615-8510, Japan \\ ${ }^{4}$ Department of Advanced Material Science, The University of Tokyo, Kashiwa, Chiba 277-8561, Japan \\ ${ }^{5}$ Office of Society-Academia Collaboration for Innovation, Kyoto University, Uji, Kyoto 611-0011, Japan \\ ${ }^{6}$ Graduate School of Global Environmental Studies, Kyoto University, Katsura, Nishikyo, Kyoto 615-8510, Japan
}

(Received 14 July 2017; accepted 6 September 2017; published online 25 September 2017)

\begin{abstract}
Clarification of the details of the interface structure between liquids and solids is crucial for understanding the fundamental processes of physical functions. Herein, we investigate the structure of the interface between tetraglyme and graphite and propose a model for the interface structure based on the observation of frequency-modulation atomic force microscopy in liquids. The ordering and distorted adsorption of tetraglyme on graphite were observed. It is found that tetraglyme stably adsorbs on graphite. Density functional theory calculations supported the adsorption structure. In the liquid phase, there is a layered structure of the molecular distribution with an average distance of $0.60 \mathrm{~nm}$ between layers. Published by AIP Publishing. https://doi.org/10.1063/1.4996226
\end{abstract}

\section{INTRODUCTION}

The nature of the interface between organic liquids and solid surfaces is an important factor affecting the characteristic functions, such as charge transfer at the electrodes, friction, catalysis, and biology. ${ }^{1-4}$ The molecular structure at the interface significantly influences these functions. ${ }^{1-4}$ For example, in electrochemical reactions on the electrodes, solvent molecules and ions are aligned at the electrode surface, forming electric double layers. ${ }^{5}$ The structure of the electric double layer affects the electrochemical reactions. ${ }^{5}$ However, the electric double layers have not been well investigated yet. It is evident from the example of the electric double layers that the clarification of the interface structure is essential for improving the functionality.

To understand the nature of the interface, a number of experimental observations of the interface structure have been performed. ${ }^{6-20}$ The interface between organic liquids and solid surfaces is referred to as a buried interface, and the analytical techniques for observing this interface are limited. To observe the interface between organic liquids and solids, the $\mathrm{X}$-ray and neutron techniques have been widely used. ${ }^{6-11} \mathrm{In}$ addition to these techniques, recent developments in scanning probe microscopy (SPM) have uncovered new aspects of the interface by directly observing the interface structure. ${ }^{12-17}$ Experimental techniques of SPM have been developed and employed to analyze the physical properties of solid surfaces in ultra-high-vacuum experiments. ${ }^{21-28}$ Recently, frequency-modulation atomic force microscopy (FM-AFM)

\footnotetext{
a)Author to whom correspondence should be addressed: minato.taketoshi. 5x@kyoto-u.ac.jp. Tel.: +81-75-383-7220.
}

has been developed to clarify the nature of the interface between liquids and solids. ${ }^{12-15,29-35}$ In this technique, a highly resolved lateral image of the surface structure of the interface can be produced. Additionally, by monitoring the force on a cantilever as a function of the tip-surface distance, the density distribution of molecules is reflected onto the force-distance curve. Using this technique, the structure of the liquid-solid interface has been clarified. ${ }^{12-15,29-35}$

Recently, rechargeable batteries have attracted increasing attention due to the expansion of the application to electric vehicles, factories, and storage of renewable energy. ${ }^{36-40} \mathrm{In}$ rechargeable batteries, the reaction at the interface between the electrode and electrolyte has a strong impact on the battery performance..$^{8-11,46-49}$ Lithium-ion battery is a type of a rechargeable battery that is widely used because of its high energy density. ${ }^{8-11,36-40}$ Graphite and tetraglyme (Fig. 1) are widely investigated in lithium-ion batteries as the electrode material and organic solvent, respectively. ${ }^{47-56}$ Understanding the structure of the interface between tetraglyme and graphite is essential for developing a lithium-ion battery with improved performance. However, much is still unknown about this interface due to the lack of direct observation via microscopic techniques.

In this research, we employed FM-AFM to observe the structure of the interface between tetraglyme and graphite. The adsorption structure of tetraglyme on the graphite surface has been clearly investigated using this technique. Additionally, we observed the molecular structure of the liquid phase at the interface. We found that a layered structure of tetraglyme was formed on graphite. The interface structure was also investigated by using density functional theory (DFT) calculations in order to clarify the atomic alignment at the interface. The resulting structure would be 


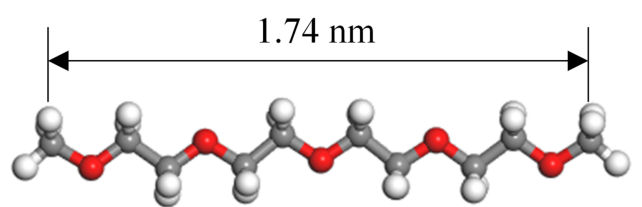

FIG. 1. Molecular structure of tetraglyme. The gray, white, and red balls represent the carbon, hydrogen, and oxygen atoms, respectively.

useful to understand the reaction at the interface between the electrolyte and electrode in rechargeable batteries, enabling the development of rechargeable batteries with improved performance.

\section{EXPERIMENTAL AND COMPUTATIONAL METHODS}

We used highly oriented pyrolytic graphite (HOPG) as the model for the graphite electrode and cleaved the HOPG (Panasonic) substrate before the experiments, which was followed by preparation of the surface by depositing $25 \mu$ l of tetraglyme (water concentration was less than $20 \mathrm{ppm}$ ) on the cleaved HOPG surface. FM-AFM experiments were performed using a microscope compatible with SPM-8000FM (Shimadzu). A silicon cantilever coated on the back with gold (PPP-NCH, Nanosensors) was used in the experiments. The oscillation frequency and amplitude of the cantilever during the experiments were $92 \mathrm{kHz}$ and $0.3 \mathrm{~nm}$, respectively. The lateral and vertical displacements of the piezoelectric scanner were calibrated using images of mica and HOPG. The observed distance in the lateral and vertical directions was averaged at least 128 data. The error bar of the measured distance was calculated from the standard deviation of the data. Throughout the experiment, the temperature was fixed at $298 \mathrm{~K}$ under Ar atmosphere to avoid dissolving the contaminants from air that affect the interface structure. $^{57}$

DFT calculations were performed in accordance with the code $\mathrm{DMol}^{3}$ of Dassault Systemes BIOVIA. ${ }^{58,59}$ The exchange-correlation energy function was represented by the Perdew-Burke-Ernzerhof (PBE) formalism. ${ }^{60}$ In the calculation, dispersion-corrected DFT (DFT-D) methods were used. The DFT-D calculations were performed using the Tkatchenko and Scheffler (TS) method. ${ }^{61}$ TS correction exploits the relationship between polarizability and volume and thus accounts to some degree for the relative variation in dispersion coefficients of differently bonded atoms. The Kohn-Sham equation was expanded in a double-numeric quality basis set (DNP) with polarization functions. Self-consistent field (SCF) procedures were performed to obtain well-converged geometrical and electronic structures at a convergence criterion of $10^{-6}$ a.u. The energy, maximum force, and maximum displacement convergence were set to $10^{-6} \mathrm{Ha}, 0.002$ $\mathrm{Ha} / \AA$, and $0.005 \AA$, respectively. The HOPG substrate is modeled with four layers of graphite. The position of the atoms at the bottom of the two layers was fixed during the optimization. The unit cell size for the calculation was $2.56 \mathrm{~nm} \times 1.97 \mathrm{~nm} \times 4.33 \mathrm{~nm}$ with $3.00 \mathrm{~nm}$ of vacuum layer. The adsorption energy of tetraglyme Ea on HOPG was calculated using the formula

$$
E_{\mathrm{a}}=E(\text { tetraglyme } / H O P G)-[E(\text { tetraglyme })+E(H O P G)],
$$

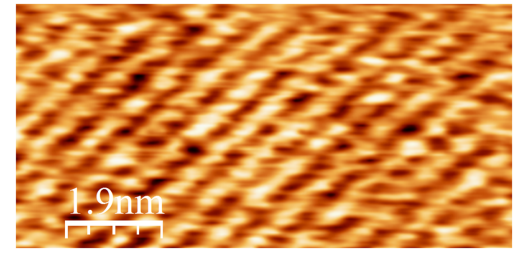

FIG. 2. The ordered structure observed in the topographic image at $\Delta f=1000$ $\mathrm{Hz}$, which was obtained in the tetraglyme/highly oriented pyrolytic graphite (HOPG) interface structure. The frequency and amplitude of the cantilever oscillation are $92 \mathrm{kHz}$ and $0.3 \mathrm{~nm}$, respectively.

where E(tetraglyme), E(HOPG), and E(tetraglyme/HOPG) are total energies of the free molecules, HOPG without adsorbates, and HOPG with adsorbed tetraglyme, respectively.

\section{RESULTS AND DISCUSSION}

The lateral structure of the interface between tetraglyme and HOPG (tetraglyme/HOPG interface) was investigated by FM-AFM. Figure 2 shows a typical topographic FM-AFM image in tetraglyme/HOPG obtained using lateral scanning. The frequency shift $\Delta f$ of the cantilever was maintained at $1000 \mathrm{~Hz}$ during scanning. The linear and ordering structure of protrusions was observed with a periodic distance of 0.62 $( \pm 0.08) \mathrm{nm}$. This image was found to be totally different from the FM-AFM image of a HOPG surface. ${ }^{62}$ This system only comprises tetraglyme and HOPG. The observed protrusions were assumed to result from the ordered structure obtained when tetraglyme was adsorbed onto the HOPG substrate. We further increased $\Delta f$ to observe the HOPG surface by approaching the cantilever; however, no different features were observed from Fig. 2. This suggests that the adsorption of tetraglyme is stable, i.e., the cantilever cannot penetrate into tetraglyme adsorbed on HOPG. Using a method proposed by Sader and Jarvis, ${ }^{63}$ the force on the tip $F$ was calculated from $\Delta f$. The calculated $F$ value for $\Delta f=1000 \mathrm{~Hz}$ in our experimental condition was approximately $700 \mathrm{pN}$. This force is higher than that in other liquids on HOPG, such as water/HOPG (less than $100 \mathrm{pN}^{61}$ ) and 1-decanol/HOPG (less than $120 \mathrm{pN}^{64}$ ) in previous reports.

In addition to the ordered structure, a distorted U-shaped protrusion (Fig. 3) was observed in the FM-AFM image with lateral scanning. The length of the protrusion is around

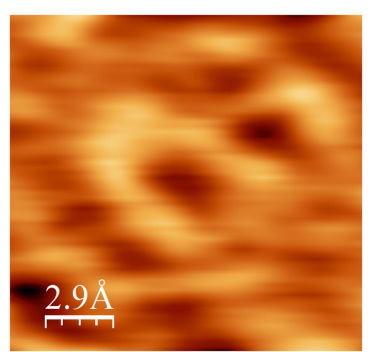

FIG. 3. A distorted structure observed in the topographic image at $\Delta f=1000$ $\mathrm{Hz}$, which was obtained in the tetraglyme/HOPG interface structure. The frequency and amplitude of the cantilever oscillation are $92 \mathrm{kHz}$ and $0.3 \mathrm{~nm}$, respectively. 
(a)

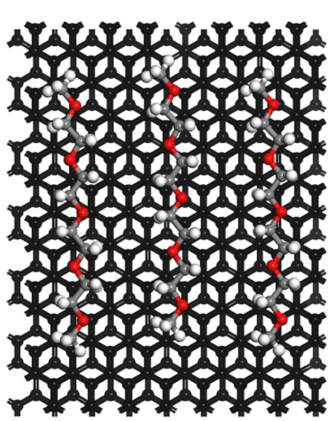

(b)

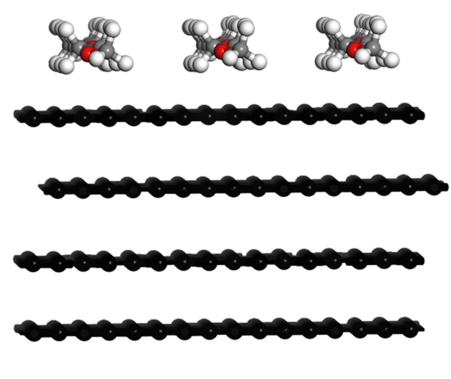

FIG. 4. Optimized adsorption structure of tetraglyme with linear structure on HOPG. (a) Top view and (b) cross-sectional view. The gray, black, white, and red balls represent carbon in tetraglyme, carbon in HOPG, hydrogen, and oxygen, respectively.

$1.70 \mathrm{~nm}$, nearly identical to that between the ends of the carbon chain in a tetraglyme molecule $(1.74 \mathrm{~nm}$ in our calculation, Fig. 1). The distorted protrusion is interpreted as tetraglyme adsorbed in a distorted structure. The distorted adsorption of tetraglyme on graphite was previously proposed by McLean et al. ${ }^{65}$

The adsorption structure of tetraglyme on HOPG was also investigated by DFT calculations. In the unit cell of the calculation, three tetraglyme molecules are placed on an area of $2.56 \mathrm{~nm} \times 1.97 \mathrm{~nm}$. Figure 4 shows the optimized adsorption structure of the system containing tetraglyme adsorbed on HOPG which is determined by the DFT calculations. The average distance between each tetraglyme molecule in the structure is $0.65 \mathrm{~nm}$, which is close to the distance between the protrusions observed in the lateral FM-AFM image (0.62 $\mathrm{nm}$ in Fig. 2). This supports the interpretation that the protrusion in the FM-AFM image (Fig. 2) is caused by tetraglyme molecules adsorbed on HOPG. The calculated adsorption energy of one tetraglyme molecule was estimated to be $1.80 \mathrm{eV}$. The adsorption energy is higher than that reported for other linear organic molecules on carbon materials $(0.21-$ $\left.0.55 \mathrm{eV}^{66}\right)$, suggesting a stable adsorption of tetraglyme on HOPG. The stable adsorption in DFT calculations matches well with the experimentally observed stable adsorption (observed a higher $F$ value than others) of tetraglyme on HOPG.

We also investigated the distorted adsorption of tetraglyme on HOPG by DFT calculations. Figure 5 shows the optimized structure of a tetraglyme molecule adsorbed on HOPG with a distorted structure. This structure was obtained with an adsorption energy of $1.78 \mathrm{eV}$. The calculated structure

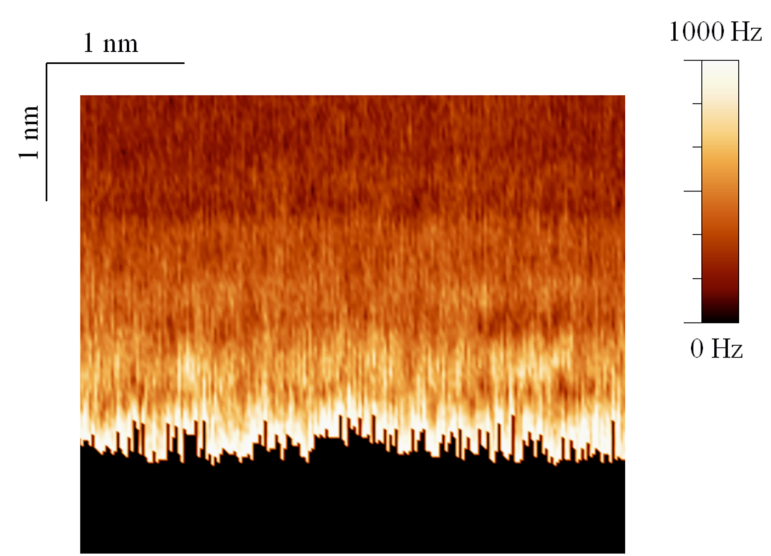

FIG. 6. Cross-sectional $\Delta f$ distribution in the tetraglyme/HOPG interface structure. The amplitude of the cantilever oscillation is $0.3 \mathrm{~nm}$. The cantilever approached the surface until $\Delta f$ reached $1000 \mathrm{~Hz}$.

of tetraglyme on HOPG is similar to the structure of the protrusion in FM-AFM (Fig. 3), suggesting that the protrusion in the FM-AFM image is caused when a single tetraglyme molecule was adsorbed on HOPG.

To analyze the molecular structure around the interface in the liquid phase, the $\Delta f$ value of the cantilever oscillation with cross-sectional scan was monitored. The oscillating cantilever was vertically scanned from the bulk of the liquid to HOPG until $\Delta f$ reached to $1000 \mathrm{~Hz}$. When $\Delta f=1000 \mathrm{~Hz}$, the cantilever was retracted from the surface toward the liquid bulk. The limits of $\Delta f$ equals the set point of lateral scanning, i.e., the laterally scanned surface (Figs. 2 and 3) corresponds to zero distance in vertical scanning. The $\Delta f$ value was recorded as a function of the vertical coordinate. By repeating the vertical scans, a $\Delta f$ distribution around the interface was obtained. A positive shift represents a repulsive force on the cantilever.

Figure 6 shows the vertical distribution of $\Delta f$ during the retraction of the cantilever to the liquid bulk. The positive $\Delta f$ value is shown in the bright area in Fig. 6. The modulation of $\Delta f$ in the liquid was observed. Four bright layers were observed in the image. The alternate appearance of the bright and dark layers indicates the formation of a layered structure, which is caused by tetraglyme in the liquid phase. On average, the distance between the bright rows is $0.60 \mathrm{~nm}$. The $\Delta f$ modulation in the vertical scan showed the same tendency when the cantilever retracted from and approached the surface (Fig. S1 of the supplementary material), implying that the observed behavior was not caused by the scanning of the cantilever. (a)

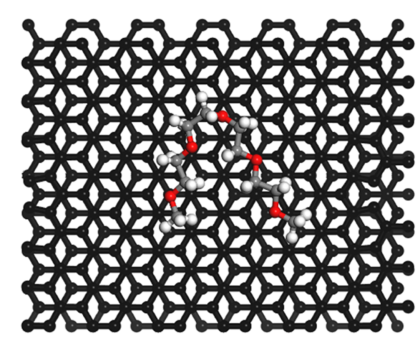

(b)
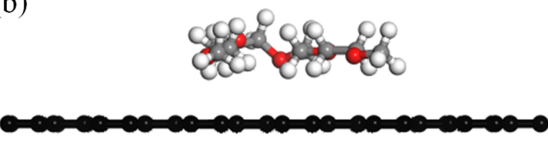

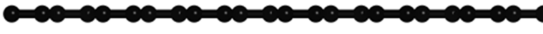

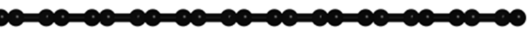

FIG. 5. Optimized adsorption structure of distorted tetraglyme on HOPG. (a) Top view and (b) cross-sectional view. The gray, black, white, and red balls represent carbon in tetraglyme, carbon in HOPG, hydrogen, and oxygen, respectively. 
(a)

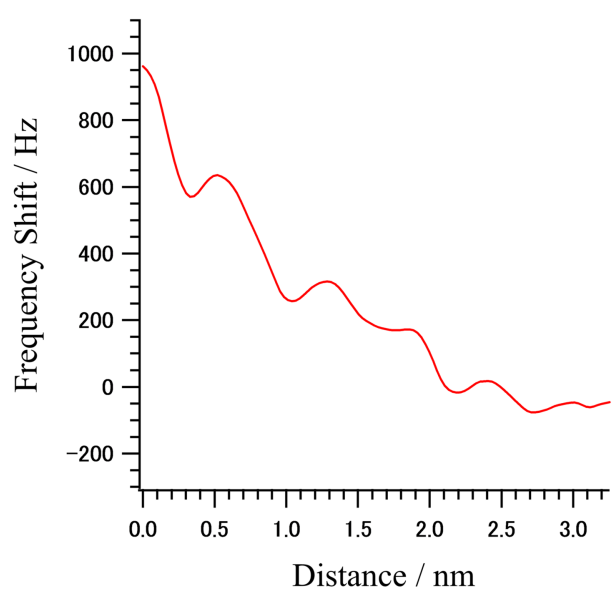

(b)

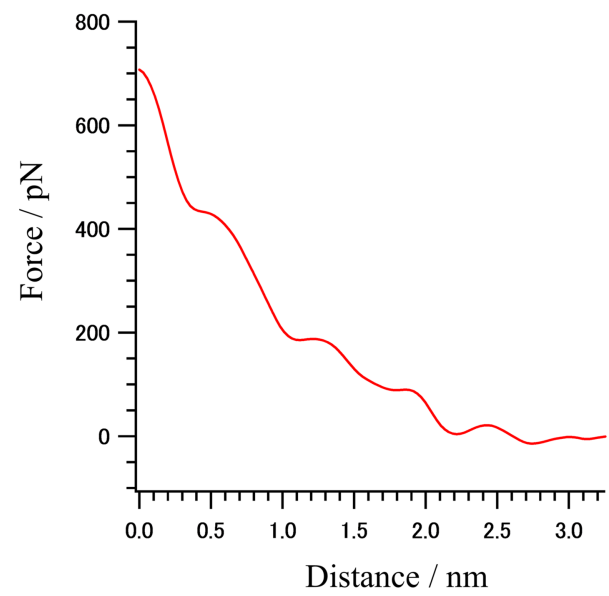

FIG. 7. Averaged vertical $\Delta f$-distance (a) and converted force-distance (b) curves obtained in the tetraglyme/HOPG interface structure. The amplitude of the cantilever oscillation was $0.3 \mathrm{~nm}$. The cantilever approached the surface until $\Delta f$ reached $1000 \mathrm{~Hz}$.
Figure 7(a) shows the averaged $\Delta f$-distance curve in vertical scanning. In addition to the three peaks observed in the cross-sectional image (Fig. 6), more peaks were observed in the curve. The average distance between the peaks was $0.60( \pm 0.09) \mathrm{nm}$, which matches well with the distance in the layer structure in Fig. 6. The experimentally observed $\Delta f$ value was converted to $F$ using a method proposed by Sader and Jarvis. ${ }^{63}$ Figure 7(b) shows the converted $F$ distance curve. Similar to $\Delta f$, the $F$ curve showed peaks and troughs that were $0.60 \mathrm{~nm}$ apart. Assuming that the tip is coated with solvent molecules using a statisticalmechanical approach, we conclude that the increased density of the molecules results in a greater force to the cantilever. ${ }^{67}$ The peaks in $\Delta f$ would be due to the high-density tetraglyme molecules. The vertical $\Delta f$ observation showed that tetraglyme molecules exist in a layered structure on the HOPG surface with an interlayer distance of $0.60 \mathrm{~nm}$ on average.

Based on the FM-AFM images obtained via lateral and vertical scanning and DFT calculations, we proposed a model for the structure at the tetraglyme/HOPG interface, as shown in Figs. 8(a) and 8(b). Tetraglyme molecules were found to adsorb on the HOPG surface with a linearly ordered structure. Moreover, the adsorption of tetraglyme on HOPG was found to be stable. In addition, when tetraglyme was adsorbed on HOPG, its structure was distorted (Figs. 3 and 5). In the liquid phase, the existing probability of tetraglyme molecules has a layered structure around the interface with an average repeat distance of $0.60 \mathrm{~nm}$. The average width of the tetraglyme layer was nearly identical to that found in previous reports, which had a layer distance of 1-decanol on HOPG. ${ }^{62}$ This suggested that the van der Waals forces between the linear chains in the molecules determine the distance of the layered structure.

As described above, the FM-AFM measurements and DFT calculations suggest that tetraglyme molecules are stably adsorbed onto HOPG. This suggests that tetraglyme would also stably adsorb on a graphite electrode in a lithiumion battery. The electrolyte-electrode interface has a strong impact on the battery performance..$^{8-11,36,37,41-50,68-70}$ The influence of the adsorbates or coating materials on the performance of a graphite electrode in lithium-ion battery is well known. ${ }^{47-49,53-55,71,72}$ If tetraglyme (or layered materials caused by the decomposition of tetraglyme) adsorbed on graphite could smoothly pass lithium ions, it would improve the cyclic performance of the battery as a protection layer. However, if tetraglyme adsorbed on graphite does not pass lithium ions, it would suppress the battery performance. Although the surface structure of a graphite electrode in a lithium-ion battery is expected to have an edge plane that is different from the surface of HOPG in this study, the adsorption of tetraglyme on the edge plane is expected to show more stability than adsorption onto the basal surface of HOPG. This is because the edge plane generally has a higher reactivity toward an organic electrolyte than the basal plane. ${ }^{73}$ Thus, a significant influence on the battery performance would be expected on the edge plane of the graphite electrode. (a)

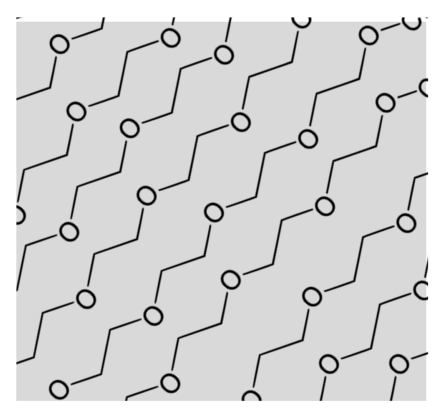

(b)

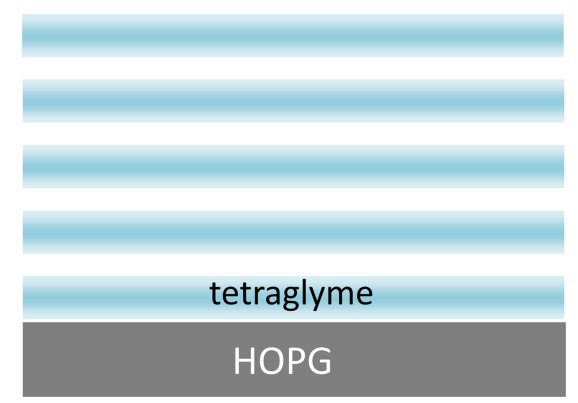

FIG. 8. Schematics of the (a) top structure and (b) crosssectional structure at the tetraglyme/HOPG interface. The molecular structure of tetraglyme is simplified for clarity. 


\section{CONCLUSIONS}

The interface structure of tetraglyme/HOPG was investigated using FM-AFM and DFT calculations, and the distorted adsorption of tetraglyme was clarified. The adsorbed tetraglyme forms a linear and ordered structure on HOPG. The FM-AFM experiments and DFT calculations suggest that the adsorption of tetraglyme on HOPG is stable. In addition, the tetraglyme in the liquid phase around the interface forms a layered structure with an average separation of $0.60 \mathrm{~nm}$. These new insights will contribute to the development of lithium-ion batteries.

\section{SUPPLEMENTARY MATERIAL}

See supplementary material for the averaged vertical $\Delta f$-distance obtained in tetraglyme/HOPG when the cantilever is approaching and retracting from the surface, respectively.

\section{ACKNOWLEDGMENTS}

This research was supported by the Research and Development Initiative for Scientific Innovation of New Generation Batteries (RISING) and Research and Development Initiative for Scientific Innovation of New Generation Batteries 2 (RISING2) projects of the New Energy and Industrial Technology Development Organization (NEDO), Japan. The liquid cell used in FM-AFM measurements was made by Mr. Yasuhiko Tsuruta (Kyoto Seisakusho Co., Ltd.). Computation time was provided by the Super Computer System, Institute for Chemical Research, Kyoto University.

${ }^{1}$ J. N. Israelachvili, Intermolecular and Surface Forces (Academic Press, London, 2011).

${ }^{2}$ M. Hugelmann, P. Hugelmann, W. Lorentz, and W. Schindler, Surf. Sci. 597, 156 (2005).

${ }^{3}$ G. M. Whitesides and B. Grzybowski, Science 295, 2418 (2002).

${ }^{4}$ H. Frauenfelder, P. W. Fenimore, G. Chen, and B. H. McMahon, Proc. Natl. Acad. Sci. U. S. A. 103, 15469 (2006).

${ }^{5}$ A. J. Bard and L. R. Faulkner, Electrochemical Methods: Fundamentals and Applications (John Wiley \& Sons, Inc., 2000).

${ }^{6}$ T. Shiarasawa, W. Voegeli, E. Arakawa, T. Takahashi, and T. Matsushita, J. Phys. Chem. C 120, 29107 (2016).

${ }^{7}$ P. Fenter and N. C. Sturchio, Prog. Surf. Sci. 77, 171 (2004).

${ }^{8}$ T. Minato et al., J. Phys. Chem. C 120, 20082 (2016).

${ }^{9}$ K. Yamamoto et al., J. Phys. Chem. C 118, 9538 (2014).

${ }^{10}$ D. Takamatsu et al., J. Phys. Chem. C 119, 9791 (2015).

${ }^{11}$ J. E. Owejan, J. P. Owejan, S. C. DeCaluwe, and J. A. Dura, Chem. Mater. 24, 2133 (2012)

${ }^{12}$ S. Morita, F. J. Giessibl, E. Meyer, and R. Wiesendanger, Noncontact Atomic Force Microscopy (Springer, 2015), Vol. 3.

${ }^{13}$ S. Ido, H. Kimiya, K. Kobayashi, H. Kominami, K. Matsushige, and H. Yamada, Nat. Mater. 13, 264 (2014).

${ }^{14}$ K. Kobayashi, H. Yamada, and K. Matsushige, Rev. Sci. Instrum. 80, 043708 (2009).

${ }^{15}$ K. I. Fukui, Y. Yokota, and A. Imanishi, Chem. Rec. 14, 964 (2014).

${ }^{16}$ K. Itaya, Prog. Surf. Sci. 58, 121 (1998).

${ }^{17}$ M. Osawa, K. Komatsu, G. Samjeske, T. Uchida, T. Ikeshoji, A. Cuesta, and C. Gutierrez, Angew. Chem., Int. Ed. 50, 1159 (2011).

${ }^{18}$ K. Motobayashi, K. Minami, N. Nishi, T. Sakka, and M. Osawa, J. Phys. Chem. Lett. 4, 3110 (2013).

${ }^{19}$ S. Nihonyanagi, S. Yamaguchi, and T. Tahara, J. Chem. Phys. 130, 204704 (2009).

${ }^{20} \mathrm{~S}$. Ye, T. Kondo, N. Hoshi, J. Inukai, S. Yoshimoto, M. Osawa, and K. Itaya, Electrochemistry 77, 2 (2009).
${ }^{21}$ G. Binning, H. Rohrer, C. Gerber, and E. Weibel, Phys. Rev. Lett. 49, 57 (1982).

${ }^{22}$ G. Binning, C. F. Quate, and C. Gerber, Phys. Rev. Lett. 56, 930 (1986).

${ }^{23}$ D. M. Eigler and E. K. Schweizer, Nature 344, 524 (1990).

${ }^{24}$ B. C. Stipe, M. A. Rezaei, and W. Ho, Science 280, 1732 (1998).

${ }^{25}$ Y. Kim, K. Motobayashi, T. Frederiksen, H. Ueba, and M. Kawai, Prog. Surf. Sci. 90, 85 (2015).

${ }^{26}$ H. Onishi and Y. Iwasawa, Phys. Rev. Lett. 76, 791 (1996).

${ }^{27}$ T. Minato, Chem. Rec. 14, 923 (2014).

${ }^{28}$ T. Minato, S. Kajita, C. L. Pang, N. Asao, Y. Yamamoto, T. Nakayama, M. Kawai, and Y. Kim, ACS Nano 9, 6837-6842 (2015).

${ }^{29}$ T. Fukuma, Y. Ueda, S. Yoshioka, and H. Asakawa, Phys. Rev. Lett. 104, 016101 (2010).

${ }^{30}$ H. Söngen, C. Marutschke, P. Spijker, E. Holmgren, I. Hermes, R. Bechstein, S. Klassen, J. Tracey, A. S. Foster, and A. Kühnle, Langmuir 33, 125 (2017).

${ }^{31}$ I. Schlesinger, K. Kuchuk, and U. Sivan, Rev. Sci. Instrum. 86, 083705 (2015).

${ }^{32}$ J. M. Black, M. B. Okatan, G. Feng, P. T. Cummings, S. V. Kalinin, and N. Balke, Nano Energy 15, 737 (2015).

${ }^{33}$ T. Arai, M. Koshioka, K. Abe, M. Tomitori, R. Kokawa, M. Ohta, H. Yamada, K. Kobayashi, and N. Oyabu, Langmuir 31, 3876 (2015).

${ }^{34}$ P. Spijker, T. Hiasa, T. Musso, R. Nishioka, H. Onishi, and A. S. Foster, J. Phys. Chem. C 118, 2058 (2014).

${ }^{35}$ T. Ichii, M. Negami, and H. Sugimura, J. Phys. Chem. C 118, 26803 (2014).

${ }^{36}$ J. M. Tarascon and M. Armand, Nature 414, 359 (2011).

${ }^{37}$ M. Armand and J. M. Tarascon, Nature 451, 652 (2008).

${ }^{38}$ R. Wagner, N. Preschitschek, S. Passerini, J. Leker, and M. Winter, J. Appl. Electrochem. 43, 481 (2013).

${ }^{39}$ V. Etacheri, R. Marom, R. Elazari, G. Salitra, and D. Aurbach, Energy Environ. Sci. 4, 3243 (2011).

${ }^{40}$ S. W. Lee, N. Yabuuchi, B. M. Gallant, S. Chen, B.-S. Kim, P. T. Hammond, and Y. Shao-Horn, Nat. Nanotechnol. 5, 531 (2010).

${ }^{41}$ D. Aurbach, J. Power Sources 89, 206 (2000).

${ }^{42}$ L. Dahéron, R. Dedryvère, H. Martinez, M. Ménétrier, C. Denage, C. Delmas, and D. Gonbeau, Chem. Mater. 20, 583 (2008).

${ }^{43}$ R. Dedryvère, M. Maccario, L. Croguennec, F. Le Cras, C. Delmas, and D. Gonbeau, Chem. Mater. 20, 7164 (2008).

${ }^{44}$ K. Xu, Chem. Rev. 114, 11503 (2014).

${ }^{45}$ M. Gauthier, T. J. Carney, A. Grimaud, L. Giordano, N. Pour, H.-H. Chang, D. P. Fenning, S. F. Lux, O. Paschos, C. Bauer, F. Maglia, S. Lupart, P. Lamp, and Y. Shao-Horn, J. Phys. Chem. Lett. 6, 4653 (2015).

${ }^{46}$ K. Iwaya, T. Ogawa, T. Minato, K. Miyoshi, J. Takeuchi, A. Kuwabara, H. Moriwake, Y. Kim, and T. Hitosugi, Phy. Rev. Lett. 111, 126104 (2013).

${ }^{47}$ R. Mogi, M. Inaba, S.-K. Jeong, Y. Iriyama, T. Abe, and Z. Ogumia, J. Electrochem. Soc. 149, A1578 (2002).

${ }^{48}$ S.-K. Jeong, M. Inaba, R. Mogi, Y. Iriyama, T. Abe, and Z. Ogumi, Langmuir 17, 8281 (2001).

${ }^{49}$ T. Abe, H. Fukuda, Y. Iriyama, and Z. Ogumi, J. Electrochem. Soc. 151, A1120 (2004).

${ }^{50}$ K. Xu, Chem. Rev. 104, 4303-4417 (2004).

${ }^{51}$ I. Hasa, X. Dou, D. Buchholz, Y. Shao-Horn, J. Hassoun, S. Passerini, and B. Scrosati, J. Power Sources 310, 26 (2016).

${ }^{52}$ S. F. Lux, M. Schmuck, G. B. Appetecchi, S. Passerini, M. Winter, and A. Balduccia, J. Power Sources 192, 606 (2009).

${ }^{53}$ T. Abe, Y. Mizutani, T. Tabuchi, K. Ikeda, M. Asano, T. Harada, M. Inaba, and Z. Ogumi, J. Power Sources 68, 216 (1997).

${ }^{54}$ S. K. Jeong, M. Inaba, Y. Iriyama, T. Abe, and Z. Ogumi, J. Power Sources 175, 540 (2008).

${ }^{55}$ K. Yoshida, M. Nakamura, Y. Kazue, N. Tachikawa, S. Tsuzuki, S. Seki, K. Dokko, and M. Watanabe, J. Am. Soc. Chem. 133, 13121 (2011).

${ }^{56}$ Y. Yamada and A. Yamada, J. Electrochem. Soc. 162, A2406 (2015).

${ }^{57}$ H. Peng, G. R. Birkett, and A. V. Nguyen, Langmuir 29, 15266 (2013).

${ }^{58}$ B. Delley, J. Chem. Phys. 92, 508 (1990).

${ }^{59}$ B. Delley, J. Chem. Phys. 113, 7756 (2000).

${ }^{60}$ J. P. Perdew, K. Burke, and M. Ernzerhof, Phys. Rev. Lett. 77, 3865 (1996).

${ }^{61}$ A. Tkatchenko and M. Scheffler, Phys. Rev. Lett. 102, 073005 (2009).

${ }^{62}$ K. Suzuki, N. Oyabu, K. Kobayashi, K. Matsushige, and H. Yamada, Appl. Phys. Express 4, 125102 (2011).

${ }^{63}$ J. E. Sader and S. P. Jarvis, Appl. Phys. Lett. 84, 1801 (2004).

${ }^{64}$ T. Hiasa, K. Kimura, and H. Onishi, J. Phys. Chem. C 116, 26475 (2012).

${ }^{65}$ B. McLean, H. Li, R. Stefanovic, R. J. Wood, G. B. Webber, K. Ueno, M. Watanabe, G. G. Warr, A. Pagea, and R. Atkin, Phys. Chem. Chem. Phys. 17, 325 (2015). 
${ }^{66}$ T. R. Rybolt, C. E. Wells, C. R. Sisson, C. B. Black, and K. A. Ziegler, J. Colloid Interface Sci. 314, 434 (2007).

${ }^{67}$ K. I. Amano, K. Suzuki, T. Fukuma, O. Takahashi, and H. Onishi, J. Chem. Phys. 139, 224710 (2013).

${ }^{68} \mathrm{~K}$. Yamamoto et al., Electrochemistry 82, 891 (2014).

${ }^{69}$ Y. Orikasa et al., Adv. Mater. Interfaces 1, 1400195 (2014).
${ }^{70}$ S. Taminato, M. Hirayama, K. Suzuki, K. Tamura, T. Minato, H. Arai, Y. Uchimoto, Z. Ogumi, and R. Kanno, J. Power Sources 307, 599 (2016).

${ }^{71}$ J. B. Goodenough and Y. Kim, Chem. Mater. 22, 587 (2010).

${ }^{72}$ P. Verma, P. Maire, and P. Novak, Electrochim. Acta 55, 6332 (2010).

${ }^{73}$ S. J. An, J. Li, C. Daniel, D. Mohanty, S. Nagpure, and D. L. Wood, Carbon 105, 52 (2016). 